\title{
Algorithmic Information, Plane Kakeya Sets, and Conditional Dimension
}

\author{
JACK H. LUTZ, Iowa State University \\ NEIL LUTZ, University of Pennsylvania
}

We formulate the conditional Kolmogorov complexity of $x$ given $y$ at precision $r$, where $x$ and $y$ are points in Euclidean spaces and $r$ is a natural number. We demonstrate the utility of this notion in two ways;

(1) We prove a point-to-set principle that enables one to use the (relativized, constructive) dimension of a single point in a set $E$ in a Euclidean space to establish a lower bound on the (classical) Hausdorff dimension of $E$. We then use this principle, together with conditional Kolmogorov complexity in Euclidean spaces, to give a new proof of the known, two-dimensional case of the Kakeya conjecture. This theorem of geometric measure theory, proved by Davies in 1971, says that every plane set containing a unit line segment in every direction has Hausdorff dimension 2.

(2) We use conditional Kolmogorov complexity in Euclidean spaces to develop the lower and upper conditional dimensions $\operatorname{dim}(x \mid y)$ and $\operatorname{Dim}(x \mid y)$ of $x$ given $y$, where $x$ and $y$ are points in Euclidean spaces. Intuitively, these are the lower and upper asymptotic algorithmic information densities of $x$ conditioned on the information in $y$. We prove that these conditional dimensions are robust and that they have the correct information-theoretic relationships with the well-studied $\operatorname{dimensions} \operatorname{dim}(x)$ and $\operatorname{Dim}(x)$ and the mutual dimensions $\operatorname{mdim}(x: y)$ and $\operatorname{Mdim}(x: y)$.

CCS Concepts: • Theory of computation $\rightarrow$ Computability; Complexity theory and logic;

Additional Key Words and Phrases: Effective dimension, Kakeya sets, Kolmogorov complexity

ACM Reference format:

Jack H. Lutz and Neil Lutz. 2018. Algorithmic Information, Plane Kakeya Sets, and Conditional Dimension. ACM Trans. Comput. Theory 10, 2, Article 7 (May 2018), 22 pages.

https://doi.org/10.1145/3201783

\section{INTRODUCTION}

This article concerns the fine-scale geometry of algorithmic information in Euclidean spaces. It shows how new ideas in algorithmic information theory can shed new light on old problems in geometric measure theory. This introduction explains these new ideas, a general principle for

A preliminary version of some of this work was published in Proceedings of the 34th International Symposium on Theoretical Aspects of Computer Science (STACS'17).

J. H. Lutz was supported in part by National Science Foundation grants 1247051 and 1545028. Some of this work was conducted at Rutgers University, DIMACS, and the Hebrew University of Jerusalem. N. Lutz was supported in part by National Science Foundation grants 1445755 and 1101690.

Authors' addresses: J. H. Lutz, Iowa State University, Department of Computer Science, 226 Atanasoff Hall, 2434 Osborn Drive, Ames, IA 50011; email: lutz@cs.iastate.edu; N. Lutz, University of Pennsylvania, Department of Computer and Information Science, Levine Hall, 3330 Walnut Street, Philadelphia, PA 19104; email: nlutz@cis.upenn.edu.

Permission to make digital or hard copies of all or part of this work for personal or classroom use is granted without fee provided that copies are not made or distributed for profit or commercial advantage and that copies bear this notice and the full citation on the first page. Copyrights for components of this work owned by others than ACM must be honored. Abstracting with credit is permitted. To copy otherwise, or republish, to post on servers or to redistribute to lists, requires prior specific permission and/or a fee. Request permissions from permissions@acm.org

(c) 2018 ACM 1942-3454/2018/05-ART7 \$15.00

https://doi.org/10.1145/3201783 
applying these ideas to classical problems, and an example of such an application. It also describes a newer concept in algorithmic information theory that arises naturally from this work.

Roughly 15 years after the mid-20th-century development of the Shannon information theory [30] of probability spaces, Kolmogorov [17] recognized that Turing's mathematical theory of computation could be used to refine the Shannon theory to enable the amount of information in individual data objects to be quantified. The resulting theory of Kolmogorov complexity, or algorithmic information theory, is now a large enterprise with many applications in computer science, mathematics, and other sciences [20]. Kolmogorov [17] proved the first version of the fundamental relationship between the Shannon and algorithmic theories of information, and this relationship was made exquisitely precise by Levin's coding theorem $[18,19]$. (Solmonoff $[31]$ and Chaitin [6, 7] independently developed Kolmogorov complexity at around the same time as Kolmogorov with somewhat different motivations.)

At the turn of the present century, the first author [21, 22] recognized that Hausdorff's [16] theory of fractal dimension is an older theory of information that can also be refined using Turing's mathematical theory of computation, thereby enabling the density of information in individual infinite data objects, such as infinite binary sequences or points in Euclidean spaces, to be quantified. The resulting theory of effective fractal dimensions is now an active enterprise with a growing array of applications [11]. The article [22] proved a relationship between effective fractal dimensions and Kolmogorov complexity that is as precise as-and uses-Levin's coding theorem.

Most of the work on effective fractal dimensions to date has concerned the (constructive) dimension $\operatorname{dim}(x)$ and the dual strong (constructive) dimension $\operatorname{Dim}(x)$ [1] of an infinite data object $x$, which for purposes of the present article is a point in a Euclidean space $\mathbb{R}^{n}$ for some positive integer $n \cdot{ }^{1}$ The inequalities

$$
0 \leq \operatorname{dim}(x) \leq \operatorname{Dim}(x) \leq n
$$

hold generally, with, for example, $\operatorname{Dim}(x)=0$ for points $x$ that are computable and $\operatorname{dim}(x)=n$ for points that are algorithmically random in the sense of Martin-Löf [27].

How can the dimensions of individual points-dimensions that are defined using the theory of computing-have any bearing on classical problems of geometric measure theory? The problems that we have in mind here are problems in which one seeks to establish lower bounds on the classical Hausdorff dimensions $\operatorname{dim}_{H}(E)$ (or other fractal dimensions) of sets $E$ in Euclidean spaces. Such problems involve global properties of sets and make no mention of algorithms.

The key to bridging this gap is relativization. Specifically, we prove here a point-to-set principle saying that to prove a lower bound $\operatorname{dim}_{H}(E) \geq \alpha$, it suffices to show that, for every $A \subseteq \mathbb{N}$ and every $\varepsilon>0$, there is a point $x \in E$ such that $\operatorname{dim}^{A}(x) \geq \alpha-\varepsilon$, where $\operatorname{dim}^{A}(x)$ is the $\operatorname{dimension}$ of $x$ relative to the oracle $A$. We also prove the analogous point-to-set principle for the classical packing dimension $\operatorname{dim}_{P}(E)$ and the relativized strong dimension $\operatorname{Dim}^{A}(x)$.

We illustrate the power of the point-to-set principle by using it to give a new proof of a known theorem in geometric measure theory. A Kakeya set in a Euclidean space $\mathbb{R}^{n}$ is a set $K \subseteq \mathbb{R}^{n}$ that contains a unit line segment in every direction. Besicovitch $[2,3]$ proved that Kakeya sets can have Lebesgue measure 0 and asked whether Kakeya sets in the Euclidean plane can have dimension less than 2 [9]. The famous Kakeya conjecture asserts a negative answer to this and to the analogous question in higher dimensions, i.e., states that every Kakeya set in a Euclidean space $\mathbb{R}^{n}$ has

\footnotetext{
${ }^{1}$ These constructive dimensions are $\Sigma_{1}^{0}$ effectivizations of Hausdorff and packing dimensions [13]. Other effectivizations, e.g., computable dimensions, polynomial time dimensions, and finite-state dimensions, have been investigated, but only the constructive dimensions are discussed here.
} 
Hausdorff dimension $n .^{2}$ This conjecture holds trivially for $n=1$ and was proven by Davies [9] for $n=2$. A version of the conjecture in finite fields has been proven by Dvir [12]. For Euclidean spaces of dimension $n \geq 3$, it is an important open problem with deep connections to other problems in analysis $[33,36]$.

In this article, we use our point-to-set principle to give a new proof of Davies's theorem. This proof does not resemble the classical proof, which is not difficult but relies on Marstrand's [26] projection theorem and point-line duality. Instead of analyzing the set $K$ globally, our proof focuses on the information content of a single, judiciously chosen, point in $K$. Given a Kakeya set $K \subseteq$ $\mathbb{R}^{2}$ and an oracle $A \subseteq \mathbb{N}$, we first choose a particular line segment $L \subseteq K$ and a particular point $(x, m x+b) \in L$, where $y=m x+b$ is the equation of the line containing $L .{ }^{3}$ We then show that $\operatorname{dim}^{A}(x, m x+b) \geq 2$. By our point-to-set principle this implies that $\operatorname{dim}_{H}(K) \geq 2$.

Our proof that $\operatorname{dim}^{A}(x, m x+b) \geq 2$ requires us to formulate a concept of conditional Kolmogorov complexity in Euclidean spaces. Specifically, for points $x \in \mathbb{R}^{m}$ and $y \in \mathbb{R}^{n}$ and natural numbers $r$, we develop the conditional Kolmogorov complexity $K_{r}(x \mid y)$ of $x$ given $y$ at precision $r$. This is a "conditional version" of the Kolmogorov complexity $K_{r}(x)$ of $x$ at precision $r$ that has been used in several recent articles, e.g., References [4, 15, 24].

In addition to enabling our new proof of Davies's theorem, conditional Kolmogorov complexity in Euclidean spaces enables us to fill a gap in effective dimension theory. The fundamental quantities in Shannon information theory are the entropy (information content) $H(X)$ of a probability space $X$, the conditional entropy $H(X \mid Y)$ of a probability space $X$ given a probability space $Y$, and the mutual information (shared information) $I(X ; Y)$ between two probability spaces $X$ and $Y$ [8]. The analogous quantities in Kolmogorov complexity theory are the Kolmogorov complexity $K(u)$ of a finite data object $u$, the conditional Kolmogorov complexity $K(u \mid v)$ of a finite data object $u$ given a finite data object $v$, and the algorithmic mutual information $I(u: v)$ between two finite data objects $u$ and $v$ [20]. The above-described dimensions $\operatorname{dim}(x)$ and $\operatorname{Dim}(x)$ of a point $x$ in Euclidean space (or an infinite sequence $x$ over a finite alphabet) are analogous by limit theorems $[1,28]$ to $K(u)$ and hence to $H(X)$. Case and the first author have recently developed and investigated the mutual dimension $\operatorname{mdim}(x: y)$ and the dual strong mutual dimension $\operatorname{Mdim}(x: y)$, which are densities of the algorithmic information shared by points $x$ and $y$ in Euclidean spaces [4] or sequences $x$ and $y$ over a finite alphabet [5]. These mutual dimensions are analogous to $I(u: v)$ and $I(X ; Y)$.

What is conspicuously missing from the above account is a notion of conditional dimension. In this article, we remedy this by using conditional Kolmogorov complexity in Euclidean space to develop the conditional dimension $\operatorname{dim}(x \mid y)$ of $x$ given $y$ and its dual, the conditional strong dimension $\operatorname{Dim}(x \mid y)$ of $x$ given $y$, where $x$ and $y$ are points in Euclidean spaces. We prove that these conditional dimensions are well behaved and that they have the correct information-theoretic relationships with the previously defined dimensions and mutual dimensions. The original plan of our proof of Davies's theorem used conditional dimensions, and we developed their basic theory to that end. Our final proof of Davies's theorem does not use them, but conditional dimensions (like the conditional entropy and conditional Kolmogorov complexity that motivate them) are very likely to be useful in future investigations.

The rest of this article is organized as follows. Section 2 briefly reviews the dimensions of points in Euclidean spaces. Section 3 presents the point-to-set principles that enable us to use dimensions

\footnotetext{
${ }^{2}$ Statements of the Kakeya conjecture vary in the literature. For example, the set is sometimes required to be compact or Borel, and the dimension used may be Minkowski instead of Hausdorff. Since the Hausdorff dimension of a set is never greater than its Minkowski dimension, our formulation is at least as strong as those variations.

${ }^{3}$ One might naïvely expect that for independently random $m$ and $x$, the point $(x, m x+b)$ must be random. In fact, in every direction there is a line that contains no random point [23].
} 
of individual points to prove lower bounds on classical fractal dimensions. Section 4 develops conditional Kolmogorov complexity and conditional dimensions in Euclidean spaces. Section 5 uses the preceding two sections to give our new proof of Davies's theorem.

\section{DIMENSIONS OF POINTS IN EUCLIDEAN SPACES}

This section reviews the constructive notions of dimension and mutual dimension in Euclidean spaces. The presentation here is in terms of Kolmogorov complexity, which quantifies the number of bits of algorithmic information in a finite data object. There are several technical variants of Kolmogorov complexity, all of which give equivalent characterizations of constructive dimensions. For convenience, we use prefix Kolmogorov complexity in this article. A prefix Turing machine is a Turing machine that takes two inputs, a program $\pi \in\{0,1\}^{*}$ and a side information string $v \in\{0,1\}^{*}$, and has the property that, for each $v \in\{0,1\}^{*}$, the set PROG $(M, v)$ of all $\pi \in\{0,1\}^{*}$ such that the computation of $M(\pi, v)$ halts is a prefix set. This means that no element of $\operatorname{PROG}(M, v)$ is a prefix of any other element of $\operatorname{PROG}(M, v)$.

The conditional Kolmogorov complexity of a string $w \in\{0,1\}^{*}$ given a string $v \in\{0,1\}^{*}$ is the minimum length $K_{M}(w \mid v)=|\pi|$ of a program $\pi \in\{0,1\}^{*}$ such that $M(\pi, v)=w$. (If there is no such string $\pi$, then $K_{M}(w \mid v)=\infty$.) Intuitively, $K_{M}(w \mid v)$ is the minimum number of bits required to "describe $w$ in the language of $M$," given the side information $v$. It is well known that there is a prefix Turing machine $U$ that is universal in the sense that, for each prefix Turing machine $M$, there is a program prefix $\pi_{M} \in\{0,1\}^{*}$ such that, for all $\pi, v \in\{0,1\}^{*}, U\left(\pi_{M} \pi, v\right)=M(\pi, v)$. We fix a universal prefix Turing machine $U$ and omit it from the notation, calling $K(w \mid v)=K_{U}(w \mid v)$ the conditional Kolmogorov complexity of $w$ given $v$. The Kolmogorov complexity of a string $w \in\{0,1\}^{*}$ is then $K(w)=K(w \mid \lambda)$, where $\lambda$ is the empty string. We write $U(\pi)$ for $U(\pi, \lambda)$. The quantity $K(w)$ is also called the algorithmic information content of the string $w$.

It is clear from the above definition of universality that each prefix Turing machine $M$ has an optimality constant $c_{M}=\left|\pi_{M}\right|$ such that, for all strings $w, v \in\{0,1\}^{*}$,

$$
K(w \mid v) \leq K_{M}(w \mid v)+c_{M},
$$

and, in particular,

$$
K(w) \leq K_{M}(w)+c_{M} .
$$

Such optimality constants are used in several of the proofs in this article.

Routine coding extends the above discussion from $\{0,1\}^{*}$ to other discrete domains, so that the Kolmogorov complexities of natural numbers, rational numbers, tuples of these, and so on, are well defined up to additive constants. Detailed discussions of prefix Turing machines and Kolmogorov complexity appear in the books $[11,20,29]$ and many articles.

The definition of $K(q)$ for rational points $q$ in Euclidean space is lifted in two steps to define the dimensions of arbitrary points in Euclidean space. First, for $x \in \mathbb{R}^{n}$ and $r \in \mathbb{N}$, the Kolmogorov complexity of $x$ at precision $r$ is

$$
K_{r}(x)=\min \left\{K(q): q \in \mathbb{Q}^{n} \cap B_{2^{-r}}(x)\right\},
$$

where $B_{2^{-r}}(x)$ is the open ball with radius $2^{-r}$ and center $x$. Second, for $x \in \mathbb{R}^{n}$, the dimension and strong dimension of $x$ are

$$
\operatorname{dim}(x)=\liminf _{r \rightarrow \infty} \frac{K_{r}(x)}{r} \quad \text { and } \quad \operatorname{Dim}(x)=\limsup _{r \rightarrow \infty} \frac{K_{r}(x)}{r}
$$

respectively. ${ }^{4}$

\footnotetext{
${ }^{4}$ We note that $K_{r}(x)=K(x \uparrow r)+o(r)$, where $x \uparrow r$ is the binary expansion of $x$, truncated $r$ bits to the right of the binary point. However, it has been known since Turing's famous correction [34] that binary notation is not a suitable
} 
Intuitively, $\operatorname{dim}(x)$ and $\operatorname{Dim}(x)$ are the lower and upper asymptotic densities of the algorithmic information in $x$. These quantities were first defined in Cantor spaces using betting strategies called gales and shown to be constructive versions of classical Hausdorff and packing dimension, respectively [1,22]. These definitions were explicitly extended to Euclidean spaces in Reference [24], where the identities (2.2) were proven as a theorem. Here it is convenient to use these identities as definitions. For $x \in \mathbb{R}^{n}$, it is easy to see that

$$
0 \leq \operatorname{dim}(x) \leq \operatorname{Dim}(x) \leq n,
$$

and it is known that, for any two reals $0 \leq \alpha \leq \beta \leq n$, there exist uncountably many points $x \in \mathbb{R}^{n}$ satisfying $\operatorname{dim}(x)=\alpha$ and $\operatorname{Dim}(x)=\beta$ [1]. Applications of these dimensions in Euclidean spaces appear in References [10, 14, 15, 24, 25].

\section{FROM POINTS TO SETS}

The central message of this article is a useful point-to-set principle by which the existence of a single high-dimensional point in a set $E \subseteq \mathbb{R}^{n}$ implies that the set $E$ has high dimension.

To formulate this principle, we use relativization. All the algorithmic information concepts in Section 2 can be relativized to an arbitrary oracle $A \subseteq \mathbb{N}$ by giving the Turing machine in their definitions oracle access to $A$. Relativized Kolmogorov complexity $K_{r}^{A}(x)$ and relativized dimensions $\operatorname{dim}^{A}(x)$ and $\operatorname{Dim}^{A}(x)$ are thus well defined. Moreover, the results of Section 2 hold relative to any oracle $A$.

\subsection{Hausdorff Dimension}

We first establish the point-to-set principle for Hausdorff dimension. Let $E \subseteq \mathbb{R}^{n}$. For $\delta>0$, define $\mathcal{U}_{\delta}(E)$ to be the collection of all countable covers of $E$ by sets of positive diameter at most $\delta$. That is, for every cover $\left\{U_{i}\right\}_{i \in \mathbb{N}} \in \mathcal{U}_{\delta}(E)$, we have $E \subseteq \bigcup_{i \in \mathbb{N}} U_{i}$ and $\left|U_{i}\right| \in(0, \delta]$ for all $i \in \mathbb{N}$, where for $X \subseteq \mathbb{R}^{n},|X|=\sup _{p, q \in X}|p-q|$. For $s \geq 0$, define

$$
H_{\delta}^{s}(E)=\inf \left\{\sum_{i \in \mathbb{N}}\left|U_{i}\right|^{s}:\left\{U_{i}\right\}_{i \in \mathbb{N}} \in \mathcal{U}_{\delta}(E)\right\} .
$$

Then the $s$-dimensional Hausdorff outer measure of $E$ is

$$
H^{s}(E)=\lim _{\delta \rightarrow 0^{+}} H_{\delta}^{s}(E)
$$

and the Hausdorff dimension of $E$ is

$$
\operatorname{dim}_{H}(E)=\inf \left\{s>0: H^{s}(E)=0\right\} .
$$

More details may be found in standard texts, e.g., References [13, 32].

Theorem 1 (Point-to-Set Principle for Hausdorff Dimension). For every set $E \subseteq \mathbb{R}^{n}$,

$$
\operatorname{dim}_{H}(E)=\min _{A \subseteq \mathbb{N}} \sup _{x \in E} \operatorname{dim}^{A}(x) .
$$

Three things should be noted about this principle. First, while the left-hand side is the classical Hausdorff dimension, which is a global property of $E$ that does not involve the theory of computing, the right-hand side is a pointwise property of the set that makes essential use of relativized algorithmic information theory. Second, as the proof shows, the right-hand side is a minimum, not merely an infimum. Third, and most crucially, this principle implies that to prove a lower bound

representation for the arguments and values of computable functions on the reals. (See also Reference [35].) Hence, to make our definitions useful for further work in computable analysis, we formulate complexities and dimensions in terms of rational approximations, both here and later. 
$\operatorname{dim}_{H}(E) \geq \alpha$, it suffices to show that, for every $A \subseteq \mathbb{N}$ and every $\varepsilon>0$, there is a point $x \in E$ such that $\operatorname{dim}^{A}(x) \geq \alpha-\varepsilon .^{5}$

For the $(\geq)$ direction of this principle, we construct the minimizing oracle $A$. The oracle encodes, for a carefully chosen sequence of increasingly refined covers for $E$, the approximate locations and diameters of all cover elements. Using this oracle, a point $x \in \mathbb{R}^{n}$ can be approximated by specifying an appropriately small cover element that it belongs to, which requires an amount of information that depends on the number of similarly-sized cover elements. We use the definition of Hausdorff dimension to bound that number. The $(\leq)$ direction can be shown using results from Reference [24], but in the interest of self-containment we prove it directly.

Proof of Theorem 1. Let $E \subseteq \mathbb{R}^{n}$, and let $d=\operatorname{dim}_{H}(E)$. For every $s>d$, we have $H^{s}(E)=0$, so there is a sequence $\left\{\left\{U_{i}^{t, s}\right\}_{i \in \mathbb{N}}\right\}_{t \in \mathbb{N}}$ of countable covers of $E$ such that $\left|U_{i}^{t, s}\right| \leq 2^{-t}$ for every $i, t \in \mathbb{N}$, and for every sufficiently large $t$ we have

$$
\sum_{i \in \mathbb{N}}\left|U_{i}^{t, s}\right|^{s}<1
$$

Let $D=\mathbb{N}^{3} \times(\mathbb{Q} \cap(d, \infty))$. Our oracle $A$ encodes functions $f_{A}: D \rightarrow \mathbb{Q}^{n}$ and $g_{A}: D \rightarrow \mathbb{Q}$ such that for every $(i, t, r, s) \in D$, we have

$$
f_{A}(i, t, r, s) \in B_{2^{-r-1}}(u)
$$

for some $u \in U_{i}^{t, s}$ and

$$
\left|g_{A}(i, t, r, s)-\right| U_{i}^{t, s}||<2^{-r-4}
$$

We will show, for every $x \in E$ and rational $s>d$, that $\operatorname{dim}^{A}(x) \leq s$.

Fix $x \in E$ and $s \in \mathbb{Q} \cap(d, \infty)$. If for any $i_{0}, t_{0} \in \mathbb{N}$ we have $x \in U_{i_{0}}^{t_{0}, s}$ and $\left|U_{i_{0}}^{t_{0}, s}\right|=0$, then $U_{i_{0}}^{t_{0}, s}=$ $\{x\}$, so $f_{A}\left(i_{0}, t_{0}, r, s\right) \in B_{2^{-r}}(x)$ for every $r \in \mathbb{N}$. In this case, let $M$ be a prefix Turing machine with oracle access to $A$ such that, whenever $U(\iota)=i \in \mathbb{N}, U(\tau)=t \in \mathbb{N}, U(\rho)=r \in \mathbb{N}$, and $U(\sigma)=q \in$ $\mathbb{Q} \cap(d, \infty)$,

$$
M(\iota \tau \rho \sigma)=f_{A}(i, t, r, q) .
$$

Now for any $r \in \mathbb{N}$, let $\iota, \tau, \rho$, and $\sigma$ be witnesses to $K\left(i_{0}\right), K\left(t_{0}\right), K(r)$, and $K(s)$, respectively. Since $i_{0}, t_{0}$, and $s$ are all constant in $r$ and $|\rho|=o(r)$, we have $|\imath \tau \rho \sigma|=o(r)$. Thus, $K_{r}^{A}(x)=o(r)$, and $\operatorname{dim}^{A}(x)=0$. Hence assume that every cover element containing $x$ has positive diameter.

Fix sufficiently large $t$, and let $U_{i_{x}}^{t, s}$ be some cover element containing $x$. Let $M^{\prime}$ be a prefix Turing machine with oracle access to $A$ such that whenever $U(\kappa)=k \in \mathbb{N}, U(\tau)=\ell \in \mathbb{N}, U(\rho)=r \in \mathbb{N}$, and $U(\sigma)=q \in \mathbb{Q} \cap(d, \infty)$,

$$
M^{\prime}(\kappa \tau \rho \sigma)=f_{A}(p, \ell, r, q),
$$

where $p$ is the $k$ th index $i$ such that $g_{A}(i, t, r, q) \geq 2^{-r-3}$.

Now fix $r \geq t-1$ such that

$$
\left|U_{i_{x}}^{t, s}\right| \in\left[2^{-r-2}, 2^{-r-1}\right) .
$$

Notice that $g_{A}\left(i_{x}, t, r, s\right) \geq 2^{-r-3}$. Hence there is some $k$ such that, letting $\kappa, \tau, \rho$, and $\sigma$ be witnesses to $K(k), K(t), K(r)$, and $K(s)$, respectively,

$$
M^{\prime}(\kappa \tau \rho \sigma) \in B_{2^{-r-1}}(u),
$$

for some $u \in U_{i_{x}}^{t, s}$. Because $\left|U_{i_{x}}^{t, s}\right|<2^{-r-1}$ and $x \in U_{i_{x}}^{t, s}$, we have

$$
M^{\prime}(\kappa \tau \rho \sigma) \in B_{2^{-r}}(x) \text {. }
$$

\footnotetext{
${ }^{5}$ The $\varepsilon$ here is useful in general but is not needed in some cases, including our proof of Theorem 16 below.
} 
Thus,

$$
K_{r}^{A}(x) \leq K(k)+K(t)+K(s)+K(r)+c,
$$

where $c$ is a machine constant for $M^{\prime}$. Since $s$ is constant in $r$ and $t<r$, Observation 4 tells us that this expression is $K(k)+o(r) \leq \log (k)+o(r)$. By Equation (3.1), there are fewer than $2^{(r+4) s}$ indices $i \in \mathbb{N}$ such that

$$
\left|U_{i}^{t, s}\right| \geq 2^{-r-4}
$$

hence by Equation (3.2) there are fewer than $2^{(r+4) s}$ indices $i \in \mathbb{N}$ such that $g_{A}(i, t, r, s) \geq 2^{-r-3}$, so $\log (k)<(r+4) s$. Therefore, $K_{r}^{A}(x) \leq r s+o(r)$.

There are infinitely many such $r$, which can be seen by replacing $t$ above with $r+2$. We have shown

$$
\operatorname{dim}^{A}(x)=\liminf _{r \rightarrow \infty} \frac{K_{r}^{A}(x)}{r} \leq s,
$$

for every rational $s>d$, hence $\operatorname{dim}^{A}(x) \leq d$. It follows that

$$
\min _{A \subseteq \mathbb{N}} \sup _{x \in E} \operatorname{dim}^{A}(x) \leq d .
$$

For the other direction, assume for contradiction that there is some oracle $A$ and $d^{\prime}<d$ such that

$$
\sup _{x \in E} \operatorname{dim}^{A}(x)=d^{\prime} .
$$

Then for every $x \in E, \operatorname{dim}^{A}(x) \leq d^{\prime}$. Let $s \in\left(d^{\prime}, d\right)$. For every $r \in \mathbb{N}$, define the sets

$$
\mathcal{B}_{r}=\left\{B_{2^{-r}}(q): q \in \mathbb{Q} \text { and } K^{A}(q) \leq r s\right\}
$$

and

$$
\mathcal{W}_{r}=\bigcup_{k=r}^{\infty} \mathcal{B}_{k} .
$$

There are at most $2^{k s+1}$ balls in each $\mathcal{B}_{k}$, so for every $r \in \mathbb{N}$ and $s^{\prime} \in(s, d)$,

$$
\begin{aligned}
\sum_{W \in \mathcal{W}_{r}}|W|^{s^{\prime}} & =\sum_{k=r}^{\infty} \sum_{W \in \mathcal{B}_{k}}|W|^{s^{\prime}} \\
& \leq \sum_{k=r}^{\infty} 2^{k s+1}\left(2^{1-k}\right)^{s^{\prime}} \\
& =2^{1+s^{\prime}} \cdot \sum_{k=r}^{\infty} 2^{\left(s-s^{\prime}\right) k},
\end{aligned}
$$

which approaches 0 as $r \rightarrow \infty$. As every $\mathcal{W}_{r}$ is a cover for $E$, we have $H^{s^{\prime}}(E)=0$, so $\operatorname{dim}_{H}(E) \leq$ $s^{\prime}<d$, a contradiction.

\subsection{Packing Dimension}

We now establish the point-to-set principle for the packing dimension, $\operatorname{dim}_{P}(E)$ of a set $E \subseteq \mathbb{R}^{n}$. Packing dimension is a dual of Hausdorff dimension satisfying $\operatorname{dim}_{P}(E) \geq \operatorname{dim}_{H}(E)$, with equality for very "regular" sets $E$. We first recall the definition.

Let $E \subseteq \mathbb{R}^{n}$. For $\delta>0$, define $\mathcal{V}_{\delta}(E)$ to be the collection of all countable packings of $E$ by disjoint open balls of diameter at most $\delta$. That is, for every packing $\left\{V_{i}\right\}_{i \in \mathbb{N}} \in \mathcal{V}_{\delta}(E)$ and every $i \in \mathbb{N}$, we have $V_{i}=B_{\varepsilon_{i}}\left(x_{i}\right)$ for some $x_{i} \in E$ and $\varepsilon_{i} \in[0, \delta / 2]$. 
For $s \geq 0$, define

$$
P_{\delta}^{s}(E)=\sup \left\{\sum_{i \in \mathbb{N}}\left|V_{i}\right|^{s}:\left\{V_{i}\right\}_{i \in \mathbb{N}} \in \mathcal{V}_{\delta}(E)\right\},
$$

and let

$$
P_{0}^{S}(E)=\lim _{\delta \rightarrow 0^{+}} P_{\delta}^{S}(E)
$$

Then the $s$-dimensional packing measure of $E$ is

$$
P^{s}(E)=\inf \left\{\sum_{i \in \mathbb{N}} P_{0}^{s}\left(E_{i}\right): E \subseteq \bigcup_{i \in \mathbb{N}} E_{i}\right\},
$$

and the packing dimension of $E$ is

$$
\operatorname{dim}_{P}(E)=\inf \left\{s: P^{s}(E)=0\right\} .
$$

Theorem 2 (Point-to-Set Principle for Packing Dimension). For every set $E \subseteq \mathbb{R}^{n}$,

$$
\operatorname{dim}_{P}(E)=\min _{A \subseteq \mathbb{N}} \sup _{x \in E} \operatorname{Dim}^{A}(x) .
$$

Proof. Let $E \subseteq \mathbb{R}^{n}$, and let $d=\operatorname{dim}_{P}(E)$. For every $s>d$, we have $P^{s}(E)=0$, so there is a cover $\left\{E_{j}^{s}\right\}_{j \in \mathbb{N}}$ for $E$ such that

$$
\sum_{j \in \mathbb{N}} \lim _{\delta \rightarrow 0^{+}} P_{\delta}^{s}\left(E_{j}^{s}\right)<1
$$

For every $r, j \in \mathbb{N}$, let

$$
\left\{V_{i}^{r, s, j}\right\}_{i \in \mathbb{N}} \in \mathcal{V}_{2^{-r-2}}\left(E_{j}^{s}\right)
$$

be a maximal packing of $E_{j}^{s}$ by open balls of radius exactly $2^{-r-2}$ with centers in $\mathbb{Q}^{n}$ (and higherindexed balls of radius 0$)$.

Let $D=\mathbb{N}^{3} \times(\mathbb{Q} \cap(d, \infty))$. Our oracle $A$ encodes a function $f_{A}: D \rightarrow \mathbb{Q}^{n}$ such that for every $(i, j, r, s) \in D$, we have

$$
f_{A}(i, j, r, s) \in V_{i}^{r, s, j} .
$$

We will show, for every $x \in E$ and rational $s>d$, that $\operatorname{Dim}^{A}(x) \leq s$.

Let $M$ be a prefix Turing machine with oracle access to $A$ such that, whenever $U(\iota)=i \in \mathbb{N}$, $U(\kappa)=j \in \mathbb{N}, U(\rho)=r \in \mathbb{N}$, and $U(\sigma)=q \in \mathbb{Q} \cap(d, \infty)$,

$$
M(\iota \kappa \rho \sigma)=f_{A}(i, j, r, s) .
$$

Fix $x \in E$ and $s \in \mathbb{Q} \cap(0, \infty)$, and let $k \in \mathbb{N}$ be such that $x \in E_{k}^{s}$. Notice that by our choice of packing, for every $r \in \mathbb{N}$ there must be some $i_{r} \in \mathbb{N}$ such that

$$
V_{i_{r}}^{r, s, k} \subseteq B_{2^{-r}}(x) .
$$

Thus, for every $r \in \mathbb{N}$, letting $\iota, \kappa, \rho, \sigma$ testify to $K\left(i_{r}\right), K(k), K(r)$, and $K(s)$, respectively,

$$
\begin{aligned}
M(\iota \kappa \rho \sigma) & =f_{A}\left(i_{r}, k, r, s\right) \\
& \in V_{i_{r}}^{r, s, k} \\
& \subseteq B_{2^{-r}}(x),
\end{aligned}
$$

hence $K_{r}^{A}(x) \leq K\left(i_{r}\right)+K(k)+K(r)+K(s)+c$, where $c$ is a machine constant for $M$. Because $k$ and $s$ are constant in $r, K(r)=o(r)$, and $K\left(i_{r}\right) \leq \log i_{r}+o(r)$, we have

$$
K_{r}^{A}(x) \leq \log i_{r}+o(r) .
$$


By Equation (3.3), $\lim _{\delta \rightarrow 0^{+}} P_{\delta}^{s}\left(E_{k}^{s}\right)<1$, so there is some $R \in \mathbb{N}$ such that, for every $r>R$, $P_{2^{-r}}^{s}\left(E_{k}^{s}\right)<1$. Then for every $r>R$,

$$
\sum_{i \in \mathbb{N}}\left|V_{i}^{r, s, k}\right|^{s}<1
$$

hence there are fewer than $2^{(r+2) s}$ balls of radius $2^{-r-2}$ in the packing, and $\log i_{r}<(r+2) s$. We conclude that $K_{r}^{A}(x) \leq r s+o(r)$ for every $r>R$, so

$$
\operatorname{dim}^{A}(x)=\limsup _{r \rightarrow \infty} \frac{K_{r}^{A}(x)}{r} \leq s .
$$

Since this holds for every rational $s>d$, we have shown $\operatorname{Dim}^{A}(x) \leq d$ and thus

$$
\min _{A \subseteq \mathbb{N}} \sup _{x \in E} \operatorname{Dim}^{A}(x) \leq d .
$$

For the other direction, assume for contradiction that there is some oracle $A$ and $d^{\prime}<d$ such that

$$
\sup _{x \in E} \operatorname{Dim}^{A}(x)=d^{\prime}
$$

Then for every $x \in E, \operatorname{Dim}^{A}(x) \leq d^{\prime}$. Let $s \in\left(d^{\prime}, d\right)$. For every $k \in \mathbb{N}$, define the set

$$
C_{k}=\bigcup\left\{B_{2^{-k}}(q): q \in \mathbb{Q} \text { and } K^{A}(q) \leq k s\right\},
$$

and for every $i \in \mathbb{N}$, define

$$
E_{i}=\bigcap_{k=i}^{\infty} C_{k} .
$$

For $r \geq i$, consider any packing in $\mathcal{V}_{2^{-r}}\left(E_{i}\right)$. Let $B_{\varepsilon}(x)$ be an element of the packing, and let $k=\lceil-\log \varepsilon\rceil$. Then $k \geq r+1>i$, so $B_{\varepsilon}(x) \subseteq E_{i} \subseteq C_{k}$. In particular, $x \in C_{k}$, meaning that there is some $q \in \mathbb{Q}$ such that $K^{A}(q) \leq k s$ and $x \in B_{2^{-k}}(q)$. As $2^{-k} \leq \varepsilon$, we also have $q \in B_{\varepsilon}(x)$. Thus, every packing element of radius at least $2^{-k}$ contains a (distinct) member of the $\operatorname{set}\left\{q \in \mathbb{Q}: K^{A}(q) \leq k s\right\}$. It follows that for every $k \geq r+1$, the packing includes at most $2^{k s+1}$ elements with diameters in the range $\left[2^{1-k}, 2^{2-k}\right)$.

Now let $s^{\prime} \in(s, d)$. For every $i \in \mathbb{N}$ and $r \geq i$, we have

$$
\begin{aligned}
P_{2^{-r}}^{s^{\prime}}\left(E_{i}\right) & =\sup \left\{\sum_{j \in \mathbb{N}}\left|V_{j}\right|^{s^{\prime}}:\left\{V_{j}\right\}_{j \in \mathbb{N}} \in \mathcal{V}_{2^{-r}}\left(E_{i}\right)\right\} \\
& \leq \sum_{k=r+1}^{\infty} 2^{k s+1}\left(2^{2-k}\right)^{s^{\prime}} \\
& =2^{1+2 s^{\prime}} \cdot \sum_{k=r+1}^{\infty} 2^{\left(s-s^{\prime}\right) k} .
\end{aligned}
$$

This approaches 0 as $r \rightarrow \infty$, so $P_{0}^{s^{\prime}}\left(E_{i}\right)=0$. Observe now that

$$
E \subseteq \bigcup_{i \in \mathbb{N}} E_{i}
$$

Thus,

$$
P^{s^{\prime}}(E) \leq \sum_{i \in \mathbb{N}} P_{0}^{s^{\prime}}\left(E_{i}\right)=0,
$$

meaning that $\operatorname{dim}_{P}(E) \leq s^{\prime}<d$, a contradiction. We conclude that for every oracle $A$,

$$
\sup _{x \in E} \operatorname{Dim}^{A}(x) \geq d \text {. }
$$




\section{CONDITIONAL KOLMOGOROV COMPLEXITY AND CONDITIONAL DIMENSIONS}

We now develop conditional Kolmogorov complexity and conditional dimensions in Euclidean spaces.

For $x \in \mathbb{R}^{m}, q \in \mathbb{Q}^{n}$, and $r \in \mathbb{N}$, the conditional Kolmogorov complexity of $x$ at precision $r$ given $q$ is

$$
\hat{K}_{r}(x \mid q)=\min \left\{K(p \mid q): p \in \mathbb{Q}^{m} \cap B_{2^{-r}}(x)\right\} .
$$

For $x \in \mathbb{R}^{m}, y \in \mathbb{R}^{n}$, and $r, s \in \mathbb{N}$, the conditional Kolmogorov complexity of $x$ at precision $r$ given $y$ at precision $s$ is

$$
K_{r, s}(x \mid y)=\max \left\{\hat{K}_{r}(x \mid q): q \in \mathbb{Q}^{n} \cap B_{2^{-s}}(y)\right\} .
$$

Intuitively, the maximizing argument $q$ is the point near $y$ that is least helpful in the task of approximating $x$. Note that $K_{r, s}(x \mid y)$ is finite, because $\hat{K}_{r}(x \mid q) \leq K_{r}(x)+O(1)$. For $x \in \mathbb{R}^{m}, y \in \mathbb{R}^{n}$, and $r \in \mathbb{N}$, the conditional Kolmogorov complexity of $x$ given $y$ at precision $r$ is

$$
K_{r}(x \mid y)=K_{r, r}(x \mid y)
$$

In parallel with Equations (2.2) and (4.13), we define the lower and upper conditional dimensions of $x \in \mathbb{R}^{m}$ given $y \in \mathbb{R}^{n}$ as

$$
\operatorname{dim}(x \mid y)=\liminf _{r \rightarrow \infty} \frac{K_{r}(x \mid y)}{r} \quad \text { and } \quad \operatorname{Dim}(x \mid y)=\limsup _{r \rightarrow \infty} \frac{K_{r}(x \mid y)}{r}
$$

respectively.

The use of the same precision bound $r$ for both $x$ and $y$ in Equation (4.3) makes the definitions (4.4) appear arbitrary and "brittle." We will show in Theorem 9 that this is not the case.

In the remainder of this section, we derive basic properties of conditional Kolmogorov complexity and conditional dimensions in Euclidean spaces. In particular, we show the following:

- $K_{r, s}(x \mid y)$ is only linearly sensitive to its precision parameters $r$ and $s$ (Lemmas 7 and 8), and the definitions (4.4) are therefore robust (Theorem 9).

- The correct relationships to mutual information and mutual dimensions hold (Theorem 10 and Corollary 11).

- These quantities obey chain rules (Theorem 12 and Corollary 13).

- Conditional Kolmogorov complexity and dimension are bounded below by relative Kolmogorov complexity and dimension (Lemma 14 and Corollary 15).

In deriving these properties, we will make use of the following four observations about Kolmogorov complexity in Euclidean spaces.

ObSeRvation 3. For every open ball $B \subseteq \mathbb{R}^{m}$ of radius $2^{-r}$,

$$
B \cap 2^{-\left(r+\left\lfloor\frac{1}{2} \log m\right\rfloor+1\right)} \mathbb{Z}^{m} \neq \emptyset .
$$

For $a \in \mathbb{Z}^{m}$, let $|a|$ denote the distance from the origin to $a$.

ObServation 4. There is a constant $c_{0} \in \mathbb{N}$ such that, for all $j \in \mathbb{N}$,

$$
K(j) \leq \log (1+j)+2 \log \log (2+j)+c_{0} .
$$

ObServation 5. There is a constant $c \in \mathbb{N}$ such that, for all $a \in \mathbb{Z}^{m}$,

$$
K(a) \leq m \log (1+|a|)+\varepsilon(|a|),
$$

where $\varepsilon(t)=c+2 \log \log (2+t)$.

Observation 6. For every $r, n \in \mathbb{N}, x \in \mathbb{R}^{n}$, and $q \in \mathbb{Q}^{n}$,

$$
K_{r}(x+q)=K_{r}(x)+O(1) .
$$


Observation 4 holds by a routine technique [20]. The proofs of Observations 5 and 6 are also routine:

Proof of Observation 5. Fix a computable, nonrepeating enumeration $a_{0}, a_{1}, \ldots$ of $\mathbb{Z}^{m}$ in which tuples $a_{j}$ appear in nondecreasing order of $\left|a_{j}\right|$. Let $M$ be a prefix Turing machine such that, for all $\pi \in\{0,1\}^{*}$, if $U(\pi) \in \mathbb{N}$, then $M(\pi)=a_{U(\pi)}$. Let $c=c_{0}+c_{M}+m+\lceil 2 \log m\rceil+2$, where $c_{0}$ is as in Observation 4 and $c_{M}$ is an optimality constant for $M$.

To see that $c$ affirms Observation 5, let $a \in \mathbb{Z}^{m}$. Let $j \in \mathbb{N}$ be the index for which $a_{j}=a$, and let $\pi \in\{0,1\}^{*}$ testify to the value of $K(j)$. Then $M(\pi)=a_{U(\pi)}=a_{j}=a$, so

$$
K(a) \leq K_{M}(a)+c_{M} \leq|\pi|+c_{M}=K(j)+c_{M} .
$$

It follows by Observation 4 that

$$
K(a) \leq \log (1+j)+2 \log \log (2+j)+c+c_{M} .
$$

We thus estimate $j$.

Let $B$ be the closed ball of radius $|a|$ centered at the origin in $\mathbb{Z}^{m}$, and let $Q$ be the solid, axisparallel $m$-cube circumscribed about $B$. Let $B^{\prime}=B \cap \mathbb{Z}^{M}$ and $Q^{\prime}=Q \cap \mathbb{Z}^{m}$. Then

$$
j \leq\left|B^{\prime}\right|-1 \leq\left|Q^{\prime}\right|-1 \leq(2|a|+1)^{m}-1,
$$

so Equation (4.5) tells us that

$$
\begin{aligned}
K(a) & \leq m \log (2|a|+1)+2 \log \log \left(1+(2|a|+1)^{m}\right)+c+c_{M} \\
& \leq m \log (2|a|+2)+2 \log (m \log (2|a|+4))+c+c_{M} .
\end{aligned}
$$

Since

$$
m \log (2|a|+2)=m+m \log (1+|a|)
$$

and

$$
\begin{aligned}
\log (m \log (2|a|+4)) & =\log m+\log (1+\log (2+|a|)) \\
& \leq \log m+1+\log \log (2+|a|),
\end{aligned}
$$

it follows that $K(a) \leq m \log (1+|a|)+\varepsilon(|a|)$.

Proof of Observation 6. Let $M$ be a prefix Turing machine such that $M(\pi \kappa)=U(\pi)+U(\kappa)$ whenever $U(\pi), U(\kappa) \in \mathbb{Q}^{n}$. If $\pi$ is a witness to $K_{r}(x)$ and $\kappa$ is a witness to $q$, then $M(\pi \kappa)=p+q$ for some $p \in B_{2^{-r}}(x)$, so $M(\pi \kappa) \in B_{2^{-r}}(x+q)$. Thus,

$$
K_{r}(x+q) \leq K_{r}(x)+K(q)+c,
$$

where $c$ is a machine constant for $M$. Since $K(q)$ is constant in $r$, we have $K_{r}(x+q) \leq K_{r}(x)+O(1)$. Applying the same argument with $-q$ replacing $q$ completes the proof.

\subsection{Linear Sensitivity to Precision Parameters}

Lemma 7 (Linear Sensitivity of $\hat{K}_{r}(x \mid q)$ To $r$ ). There is a constant $c_{1} \in \mathbb{N}$ such that, for all $x \in \mathbb{R}^{m}, q \in \mathbb{Q}^{n}$, and $r, \Delta r \in \mathbb{N}$,

$$
\hat{K}_{r}(x \mid q) \leq \hat{K}_{r+\Delta r}(x \mid q) \leq \hat{K}_{r}(x \mid q)+m \Delta r+\varepsilon_{1}(r, \Delta r),
$$

where $\varepsilon_{1}(r, \Delta r)=2 \log (1+\Delta r)+K(r, \Delta r)+c_{1}$.

Proof. Let $M$ be a prefix Turing machine such that, for all $\pi_{1}, \pi_{2}, \pi_{3} \in\{0,1\}^{*}$ and $q \in \mathbb{Q}^{n}$, if $U\left(\pi_{1}, q\right)=p \in \mathbb{Q}^{m}, U\left(\pi_{2}\right)=(r, \Delta r) \in \mathbb{N}^{2}$, and $U\left(\pi_{3}\right)=a \in \mathbb{Z}^{m}$, then $M\left(\pi_{1} \pi_{2} \pi_{3}, q\right)=p+2^{-r^{*}} a$, where $r^{*}=r+\Delta r+\left\lfloor\frac{1}{2} \log m\right\rfloor+1$. Let $c_{1}=c+c_{M}+3 m+m\left\lfloor\frac{1}{2} \log m\right\rfloor+\left\lceil 2 \log \left(3+\left\lfloor\frac{1}{2} \log m\right\rfloor\right)\right\rceil$, where $c$ is the constant from Observation 5 and $c_{M}$ is an optimality constant for $M$. 
To see that $c_{1}$ affirms the lemma, let $x, q, r$, and $\Delta r$ be as given. The first inequality holds trivially. To see that the second inequality holds, let $\pi_{1}, \pi_{2} \in\{0,1\}^{*}$ testify to the values of $\hat{K}_{r}(x \mid q)$ and $K(r, \Delta r)$, respectively. Let $B=B_{2^{-r}}(x), B^{\prime}=B_{2^{-(r+\Delta r)}}(x)$, and $p=U\left(\pi_{1}, q\right)$, noting that $p \in \mathbb{Q}^{m} \cap B$. Applying Observation 3 to the ball $B^{\prime}-p$ tells us that

$$
\left(B^{\prime}-p\right) \cap 2^{-r^{*}} \mathbb{Z}^{m} \neq \emptyset,
$$

i.e., that

$$
B^{\prime} \cap\left(p+2^{-r^{*}} \mathbb{Z}^{m}\right) \neq \emptyset
$$

So fix a point $p^{\prime} \in B^{\prime} \cap\left(p+2^{-r^{*}} \mathbb{Z}^{m}\right)$, say, $p^{\prime}=p+2^{-r^{*}} a$, where $a \in \mathbb{Z}^{m}$, and let $\pi_{3} \in\{0,1\}^{*}$ testify to the value of $K(a)$. Then

$$
M\left(\pi_{1} \pi_{2} \pi_{3}, q\right)=p^{\prime} \in \mathbb{Q} \cap B^{\prime},
$$

so

$$
\begin{aligned}
\hat{K}_{r+\Delta r}(x \mid q) & \leq K\left(p^{\prime} \mid q\right) \\
& \leq \hat{K}_{M}\left(p^{\prime} \mid q\right)+c_{M} \\
& \leq\left|\pi_{1} \pi_{2} \pi_{3}\right|+c_{M} .
\end{aligned}
$$

By our choice of $\pi_{1}, \pi_{2}$, and $\pi_{3}$, this implies that

$$
\hat{K}_{r+\Delta r}(x \mid q) \leq \hat{K}_{r}(x \mid q)+K(r, \Delta r)+K(a)+c_{M} .
$$

We thus estimate $K(a)$.

Since

$$
\begin{aligned}
|a| & =2^{r^{*}}\left|p^{\prime}-p\right| \\
& \leq 2^{r^{*}}\left(\left|p^{\prime}-x\right|+|p-x|\right) \\
& <2^{r^{*}}\left(2^{-(r+\Delta r)}+2^{-r}\right) \\
& =2^{1+\left\lfloor\frac{1}{2} \log m\right\rfloor}\left(1+2^{\Delta r}\right),
\end{aligned}
$$

Observation 5 tells us that

$$
\begin{aligned}
K(a) & \leq m \log \left(1+2^{\left\lfloor\frac{1}{2} \log m\right\rfloor}\left(1+2^{\Delta r}\right)\right)+\varepsilon(|a|) \\
& \leq m \log \left(2^{\Delta r+3+\left\lfloor\frac{1}{2} \log m\right\rfloor}\right)+\varepsilon(|a|),
\end{aligned}
$$

i.e., that

$$
K(a) \leq m \Delta r+3 m+m\left\lfloor\frac{1}{2} \log m\right\rfloor+\varepsilon(|a|),
$$

where

$$
\begin{aligned}
\varepsilon(|a|) & \leq c+2 \log \log \left(2+2^{1+\left\lfloor\frac{1}{2} \log m\right\rfloor}\left(1+2^{\Delta r}\right)\right) \\
& \leq c+2 \log \log \left(2^{\Delta r+3+\left\lfloor\frac{1}{2} \log m\right\rfloor}\right) \\
& =c+2 \log \left(\Delta r+\left\lfloor\frac{1}{2} \log m\right\rfloor+3\right) \\
& \leq c+2 \log \left((1+\Delta r)\left(3+\left\lfloor\frac{1}{2} \log m\right\rfloor\right)\right) \\
& =c+2 \log (1+\Delta r)+2 \log \left(3+\left\lfloor\frac{1}{2} \log m\right\rfloor\right) .
\end{aligned}
$$

It follows by Equations (4.6) and (4.7) that

$$
\hat{K}_{r+\Delta r}(x \mid q) \leq \hat{K}_{r}(x \mid q)+m \Delta r+\varepsilon_{1}(r, \Delta r) .
$$


Lemma 8 (Linear Sensitivity of $K_{r, s}(x \mid y)$ To $s$ ). There is a constant $c_{2} \in \mathbb{N}$ such that, for all $x \in \mathbb{R}^{m}, y \in \mathbb{R}^{n}$, and $r, s, \Delta s \in \mathbb{N}$,

$$
K_{r, s}(x \mid y) \geq K_{r, s+\Delta s}(x \mid y) \geq K_{r, s}(x \mid y)-n \Delta s-\varepsilon_{2}(s, \Delta s),
$$

where $\varepsilon_{2}(s, \Delta s)=2 \log (1+\Delta s)+K(s, \Delta s)+c_{2}$.

Proof. Let $M$ be a prefix Turing machine such that, for all $\pi_{1}, \pi_{2}, \pi_{3} \in\{0,1\}^{*}$ and $q \in \mathbb{Q}^{n}$, if $U\left(\pi_{1}\right)=(s, \Delta s) \in \mathbb{N}^{2}$ and $U\left(\pi_{2}\right)=a \in \mathbb{Z}^{m}$, then $M\left(\pi_{1} \pi_{2} \pi_{3}, q\right)=U\left(\pi_{3}, q+2^{-s^{*}} a\right)$, where $s^{*}=s+$ $\Delta s+\left\lceil\frac{1}{2} \log n\right\rceil$. Let $c_{2}=c+c_{M}+3 n+n\left\lfloor\frac{1}{2} \log n\right\rfloor+2\left\lceil 2 \log \left(3+\left\lfloor\frac{1}{2} \log n\right\rfloor\right)\right\rceil$, where $c$ is the constant from Observation 5 and $c_{M}$ is an optimality constant for $M$.

To see that $c_{2}$ affirms the lemma, let $x, y, r, s$, and $\Delta s$ be as given The first inequality holds trivially. To see that the second inequality holds, let $B=B_{2^{-}}(y), B^{\prime}=B_{2^{-(s+\Delta s)}}(y)$, and $q \in \mathbb{Q}^{n} \cap B$. It suffices to prove that

$$
\hat{K}_{r}(x \mid q) \leq K_{r, s+\Delta s}(x \mid y)+n \Delta s+\varepsilon_{2}(s, \Delta s) .
$$

Let $\pi_{1} \in\{0,1\}^{*}$ testify to the value of $K(s, \Delta s)$. Applying Observation 3 to the ball $B^{\prime}-q$ tells us that

$$
\left(B^{\prime}-q\right) \cap 2^{-s^{*}} \mathbb{Z}^{n} \neq \emptyset,
$$

i.e., that

$$
B^{\prime} \cap\left(q+2^{-s^{*}} \mathbb{Z}^{n}\right) \neq \emptyset .
$$

So fix a point $q^{\prime} \in B^{\prime} \cap\left(q+2^{-s^{*}} \mathbb{Z}^{n}\right)$, say, $q^{\prime}=q+2^{-s^{*}} a$, where $a \in \mathbb{Z}^{n}$. Note that

$$
\hat{K}_{r}\left(x \mid q^{\prime}\right) \leq K_{r, s+\Delta s}(x \mid y) \text {. }
$$

Let $\pi_{2}, \pi_{3} \in\{0,1\}^{*}$ testify to the values of $K(a)$ and $\hat{K}_{r}\left(x \mid q^{\prime}\right)$, respectively, noting that $U\left(\pi_{3}, q^{\prime}\right)=p$ for some $p \in \mathbb{Q}^{m} \cap B_{2^{-r}}(x)$. Then

$$
M\left(\pi_{1} \pi_{2} \pi_{3}, q\right)=U\left(\pi_{3}, q^{\prime}\right)=p \in \mathbb{Q}^{m} \cap B_{2^{-r}}(x),
$$

so

$$
\begin{aligned}
\hat{K}_{r}(x \mid q) & \leq K(p \mid q) \\
& \leq K_{M}(p \mid q)+c_{M} \\
& \leq\left|\pi_{1} \pi_{2} \pi_{3}\right|+c_{M} .
\end{aligned}
$$

By our choice of $\pi_{1}, \pi_{2}$, and $\pi_{3}$, and by Equation (4.9), this implies that

$$
\hat{K}_{r}(x \mid q) \leq K_{r, s+\Delta s}(x \mid y)+K(a)+K(s, \Delta s)+c_{M} .
$$

We thus estimate $K(a)$.

Since

$$
\begin{aligned}
|a| & =2^{s^{*}}\left|q^{\prime}-q\right| \\
& \leq 2^{s^{*}}\left(\left|q^{\prime}-y\right|+|q-y|\right) \\
& <2^{s^{*}}\left(s^{-(s+\Delta s)}+2^{-s}\right) \\
& =2^{1+\left\lfloor\frac{1}{2} \log n\right\rfloor}\left(1+2^{\Delta s}\right),
\end{aligned}
$$

Observation 5 tells us that

$$
\begin{aligned}
K(a) & \leq n \log \left(1+2^{1+\left\lfloor\frac{1}{2} \log n\right\rfloor}\left(1+2^{\Delta s}\right)\right)+\varepsilon(|a|) \\
& \leq n \log \left(2^{\Delta s+3+\left\lfloor\frac{1}{2} \log n\right\rfloor}\right)+\varepsilon(|a|),
\end{aligned}
$$

i.e., that

$$
K(a) \leq n \Delta s+3 n+n\left\lfloor\frac{1}{2} \log n\right\rfloor+\varepsilon(|a|),
$$


where

$$
\begin{aligned}
\varepsilon(|a|) & \leq c+2 \log \log \left(2+2^{1+\left\lfloor\frac{1}{2} \log n\right\rfloor}\left(1+2^{\Delta s}\right)\right) \\
& \leq c+2 \log \log \left(2^{\Delta s+3+\left\lfloor\frac{1}{2} \log n\right\rfloor}\right) \\
& =c+2 \log \left(\Delta s+3+\left\lfloor\frac{1}{2} \log n\right\rfloor\right) \\
& \leq c+2 \log \left((1+\Delta s)\left(3+\left\lfloor\frac{1}{2} \log n\right\rfloor\right)\right) \\
& =c+2 \log (1+\Delta s)+2 \log \left(3+\left\lfloor\frac{1}{2} \log n\right\rfloor\right) .
\end{aligned}
$$

It follows by Equations (4.10) and (4.11) that Equation (4.8) holds.

Theorem 9. Let $s: \mathbb{N} \rightarrow \mathbb{N}$. If $|s(r)-r|=o(r)$, then, for all $x \in \mathbb{R}^{m}$ and $y \in \mathbb{R}^{n}$,

$$
\operatorname{dim}(x \mid y)=\liminf _{r \rightarrow \infty} \frac{K_{r, s(r)}(x \mid y)}{r}
$$

and

$$
\operatorname{Dim}(x \mid y)=\limsup _{r \rightarrow \infty} \frac{K_{r, s(r)}(x \mid y)}{r} .
$$

Proof. Assume the hypothesis. Define $s^{-}, s^{+}: \mathbb{N} \rightarrow \mathbb{N}$ by

$$
s^{-}(r)=\min \{r, s(r)\}, s^{+}(r)=\max \{r, s(r)\} .
$$

Lemma 8 tells us that, for all $x \in \mathbb{R}^{m}$ and $y \in \mathbb{R}^{n}$,

$$
\begin{aligned}
K_{r, s^{-}(r)}(x \mid y) & \geq K_{r, r}(x \mid y) \\
& \geq K_{r, s^{+}(r)}(x \mid y) \\
& \geq K_{r, s^{-}(r)}(x \mid y)-O\left(s^{+}(r)-s^{-}(r)\right)-o(r) \\
& =K_{r, s^{-}(r)}(x \mid y)-O(|s(r)-r|)-o(r) \\
& =K_{r, s^{-}(r)}(x \mid y)-o(r) .
\end{aligned}
$$

Since

it follows that

$$
K_{r, s^{-}(r)}(x \mid y) \geq K_{r, s(r)}(x \mid y) \geq K_{r, s^{+}(r)}(x \mid y)
$$

$$
\left|K_{r, s(r)}(x \mid y)-K_{r, r}(x \mid y)\right|=o(r)
$$

The theorem follows immediately.

\subsection{Relationships to Mutual Information and Dimensions}

Mutual dimensions were developed very recently, and Kolmogorov complexity was the starting point. The mutual (algorithmic) information between two strings $u, v \in\{0,1\}^{*}$ is

$$
I(u: v)=K(v)-K(v \mid u) .
$$

Again, routine coding extends $K(u \mid v)$ and $I(u: v)$ to other discrete domains. Discussions of $K(u \mid v)$, $I(u: v)$, and the correspondence of $K(u), K(u \mid v)$, and $I(u: v)$ with Shannon entropy, Shannon conditional entropy, and Shannon mutual information appear in Reference [20].

In parallel with Equations (2.1) and (2.2), Case and the first author [4] lifted the definition of $I(p: q)$ for rational points $p$ and $q$ in Euclidean spaces in two steps to define the mutual dimensions between two arbitrary points in (possibly distinct) Euclidean spaces. First, for $x \in \mathbb{R}^{m}, y \in \mathbb{R}^{n}$, and $r \in \mathbb{N}$, the mutual information between $x$ and $y$ at precision $r$ is

$$
I_{r}(x: y)=\min \left\{I(p: q): p \in B_{2^{-r}}(x) \cap \mathbb{Q}^{m} \text { and } q \in B_{2^{-r}}(y) \cap \mathbb{Q}^{n}\right\},
$$


where $B_{2^{-r}}(x)$ and $B_{2^{-r}}(y)$ are the open balls of radius $2^{-r}$ about $x$ and $y$ in their respective Euclidean spaces. Second, for $x \in \mathbb{R}^{m}$ and $y \in \mathbb{R}^{n}$, the lower and upper mutual dimensions between $x$ and $y$ are

$$
\operatorname{mdim}(x: y)=\liminf _{r \rightarrow \infty} \frac{I_{r}(x: y)}{r} \text { and } \operatorname{Mdim}(x: y)=\limsup _{r \rightarrow \infty} \frac{I_{r}(x: y)}{r},
$$

respectively. Useful properties of these mutual dimensions, especially including data processing inequalities, appear in Reference [4].

Theorem 10. For all $x \in \mathbb{R}^{m}$ and $y \in \mathbb{R}^{n}$,

$$
I_{r}(x: y)=K_{r}(x)-K_{r}(x \mid y)+o(r) .
$$

Proof. Let $B_{x}=B_{2^{-r}}(x) \cap \mathbb{Q}^{m}$ and $B_{y}=B_{2^{-r}}(y) \cap \mathbb{Q}^{n}$. Let $p_{0}$ and $q_{0}$ be $K$-minimizers for $B_{x}$ and $B_{y}$, respectively, such that

$$
I_{r}(x: y)=I\left(p_{0}: q_{0}\right)+o(r) .
$$

These exist by Theorem 4.6 of Reference [4]. Then

$$
\begin{aligned}
K_{r}(x)-K_{r}(x \mid y) & =\min _{p \in B_{x}} K(p)-\max _{q \in B_{y}} \min _{p \in B_{x}} K(p \mid q) \\
& \geq \min _{p \in B_{x}} K(p)-\min _{p \in B_{x}} \max _{q \in B_{y}} K(p \mid q) \\
& =\min _{p \in B_{x}} K(p)-\min _{p \in B_{x}} K\left(p \mid q_{0}\right)+o(r),
\end{aligned}
$$

by Lemma 4.2 and Observation 3.7 of Reference [4]. By our choice of $p_{0}$, this is equal to

$$
\begin{aligned}
K\left(p_{0}\right)-\min _{p \in B_{x}} K\left(p \mid q_{0}\right)+o(r) & \geq K\left(p_{0}\right)-K\left(p_{0} \mid q_{0}\right)+o(r) \\
& =I\left(p_{0}: q_{0}\right)+o(r) \\
& =I_{r}(x: y)+o(r) .
\end{aligned}
$$

For the other direction, let $p_{1} \in B_{x}$ be such that

$$
K\left(p_{1} \mid q_{0}\right)=\min _{p \in B_{x}} K\left(p \mid q_{0}\right) .
$$

By Lemma 4.5 of Reference [4],

$$
\begin{aligned}
I\left(p_{0}: q_{0}\right) & \geq K\left(p_{1}\right)-K\left(p_{1} \mid p_{0}, K\left(p_{0}\right)\right)-K\left(p_{1} \mid q_{0}, K\left(q_{0}\right)\right)+o(r) \\
& \geq K\left(p_{1}\right)-K\left(p_{1} \mid p_{0}, K\left(p_{0}\right)\right)-K\left(p_{1} \mid q_{0}\right)+o(r) .
\end{aligned}
$$

Now

$$
\begin{aligned}
K\left(p_{0}\right)+K\left(p_{1} \mid p_{0}, K\left(p_{0}\right)\right)+o(r) & =K\left(p_{0}, p_{1}\right) \\
& =K\left(p_{1}\right)+K\left(p_{0} \mid p_{1}, K\left(p_{1}\right)\right)+o(r) \\
& \leq K\left(p_{1}\right)+K\left(p_{0} \mid p_{1}\right)+o(r) \\
& =K\left(p_{1}\right)+o(r),
\end{aligned}
$$

by Corollary 4.4 of Reference [4]. So

$$
K\left(p_{1}\right)-K\left(p_{1} \mid p_{0}, K\left(p_{0}\right)\right) \geq K\left(p_{o}\right)+o(r) .
$$


Thus, by Equation (4.15),

$$
\begin{aligned}
I\left(p_{0}: q_{0}\right) & \geq K\left(p_{0}\right)-K\left(p_{1} \mid q_{0}\right)+o(r) \\
& =K\left(p_{0}\right)-\min _{p \in B_{x}} K\left(p \mid q_{0}\right)+o(r) \\
& =K_{r}(x)-\min _{p \in B_{x}} K\left(p \mid q_{0}\right)+o(r) \\
& \geq K_{r}(x)-\max _{q \in B_{y}} \min _{p \in B_{x}} K(p \mid q)+o(r) \\
& =K_{r}(x)-K_{r}(x \mid y)+o(r) .
\end{aligned}
$$

Then by Equation (4.14), $I_{r}(x: y) \geq K_{r}(x)-K_{r}(x \mid y)+o(r)$, so equality holds.

Corollary 11. For all $x \in \mathbb{R}^{m}$ and $y \in \mathbb{R}^{n}$, the following hold.

(1) $\operatorname{mdim}(x: y) \geq \operatorname{dim}(x)-\operatorname{Dim}(x \mid y)$.

(2) $\operatorname{Mdim}(x: y) \leq \operatorname{Dim}(x)-\operatorname{dim}(x \mid y)$.

\subsection{Chain Rules}

Theorem 12 (Chain Rule For $K_{r}$ ). For all $x \in \mathbb{R}^{m}$ and $y \in \mathbb{R}^{n}$,

$$
K_{r}(x, y)=K_{r}(x \mid y)+K_{r}(y)+o(r) .
$$

Proof. Theorem 4.10 of Reference [4] tells us that

$$
I_{r}(x: y)=K_{r}(x)+K_{r}(y)-K_{r}(x, y)+o(r) .
$$

Combining this with Theorem 10, we have

$$
K_{r}(x)+K_{r}(y)-K_{r}(x, y)+o(r)=K_{r}(x)-K_{r}(x \mid y)+o(r) .
$$

The theorem follows immediately.

Corollary 13 (Chain Rule for Dimension). For all $x \in \mathbb{R}^{m}$ and $y \in \mathbb{R}^{n}$,

$$
\begin{aligned}
\operatorname{dim}(x)+\operatorname{dim}(y \mid x) & \leq \operatorname{dim}(x, y) \\
& \leq \operatorname{dim}(x)+\operatorname{Dim}(y \mid x) \\
& \leq \operatorname{Dim}(x, y) \\
& \leq \operatorname{Dim}(x)+\operatorname{Dim}(y \mid x) .
\end{aligned}
$$

\subsection{Relationships to Relative Kolmogorov Complexity and Dimensions}

We also consider the Kolmogorov complexity of $x \in \mathbb{R}^{m}$ at precision $r$ relative to $y \in \mathbb{R}^{n}$. Let $K_{r}^{y}(x)$ denote $K_{r}^{A_{y}}(x)$, where $A_{y} \subseteq \mathbb{N}$ encodes the binary expansions of $y$ 's coordinates. The following lemma reflects the intuition that oracle access to $y$ is at least as useful as any bounded-precision estimate for $y$.

Lemma 14. For each $m, n \in \mathbb{N}$ there is a constant $c \in \mathbb{N}$ such that, for all $x \in \mathbb{R}^{m}, y \in \mathbb{R}^{n}$, and $r, s \in \mathbb{N}$,

$$
K_{r}^{y}(x) \leq K_{r, s}(x \mid y)+K(s)+c .
$$

In particular, $K_{r}^{y}(x) \leq K_{r}(x \mid y)+K(r)+c$.

Proof. Let $m, n \in \mathbb{N}$, and let $U$ be the optimal prefix Turing machine fixed for the definition of conditional Kolmogorov complexity. Let $M$ be an oracle prefix Turing machine that, on input $\pi \in$ $\{0,1\}^{*}$ with oracle $g: \mathbb{N} \rightarrow \mathbb{Q}^{n}$, does the following. If $\pi$ is of the form $\pi=\pi_{1} \pi_{2}$, where $U\left(\pi_{1}, \lambda\right)=$ $t \in \mathbb{N}$, then $M$ simulates $U\left(\pi_{2}, g(t)\right)$. Let $c$ be an optimality constant for the machine $M$. 
To see that $c$ affirms the lemma, let $x \in \mathbb{R}^{m}, y \in \mathbb{R}^{n}$, and $r, s \in \mathbb{N}$. Let $q=y\lceil(s+\log \sqrt{n})$, the truncation of the binary expansions of each of $y$ 's coordinates to $s+\log \sqrt{n}$ bits to the right of the binary point. Let $\pi_{s} \in\{0,1\}^{*}$ testify to the value of $K(s)$, and let $\pi_{x}$ testify to the value of $\hat{K}_{r}(x \mid q)$. Then

$$
q \in \mathbb{Q}^{n} \cap B_{2^{-s}}(y)
$$

and

$$
M^{y}\left(\pi_{s} \pi_{x}\right)=U\left(\pi_{x}, q\right) \in \mathbb{Q}^{m} \cap B_{2^{-r}}(x),
$$

so

$$
\begin{aligned}
K_{r}^{y}(x) & \leq K_{M, r}^{y}(x)+c \\
& \leq\left|\pi_{s} \pi_{x}\right|+c \\
& =\hat{K}_{r}(x \mid q)+K(s)+c \\
& \leq K_{r, s}(x \mid y)+K(s)+c .
\end{aligned}
$$

Corollary 15. For all $x \in \mathbb{R}^{m}$ and $y \in \mathbb{R}^{n}$,

$$
\operatorname{dim}^{y}(x) \leq \operatorname{dim}(x \mid y) \text { and } \operatorname{Dim}^{y}(x) \leq \operatorname{Dim}(x \mid y) .
$$

\section{KAKEYA SETS IN THE PLANE}

This section uses the results of the preceding two sections to give a new proof of the following classical theorem. Recall that a Kakeya set in $\mathbb{R}^{n}$ is a set containing a unit line segment in every direction.

Theorem 16 (Davies [9]). Every Kakeya set in $\mathbb{R}^{2}$ has Hausdorff dimension 2.

Our new proof of Theorem 16 uses a relativized version of the following lemma.

Lemma 17. Let $m \in[0,1]$ and $b \in \mathbb{R}$. Then for almost every $x \in[0,1]$,

$$
\liminf _{r \rightarrow \infty} \frac{K_{r}(m, b, x)-K_{r}(b \mid m)}{r} \leq \operatorname{dim}(x, m x+b) .
$$

Proof. We build a program that takes as input a precision level $r$, an approximation $p$ of $x$, an approximation $q$ of $m x+b$, a program $\pi$ that will approximate $b$ given an approximation for $m$, and a natural number $h$. In parallel, the program considers each multiple of $2^{-r}$ in $[0,1]$ as a possible approximate value $u$ for the slope $m$, and it checks whether each such $u$ is consistent with the program's inputs. If $u$ is close to $m$, then $\pi(u)$ will be close to $b$, so $u p+\pi(u)$ will be close to $m x+b$. Any $u$ that satisfies this condition is considered a "candidate" for approximating $m$.

Some of these candidates may be "false positives," in that there can be values of $u$ that are far from $m$ but for which $u p+\pi(u)$ is still close to $m x+b$. Thus, the program is also given an input $h$ so that it can choose the correct candidate; it selects the $h$ th candidate that arises in its execution. We will show that this $h$ is often not large enough to significantly affect the total input length.

Formally, let $M$ be a prefix Turing machine that runs the following algorithm on input $\rho \pi \sigma \eta$ whenever $U(\rho)=r \in \mathbb{N}, U(\eta)=h \in \mathbb{N}$, and $U(\sigma)=(p, q) \in \mathbb{Q}^{2}$.

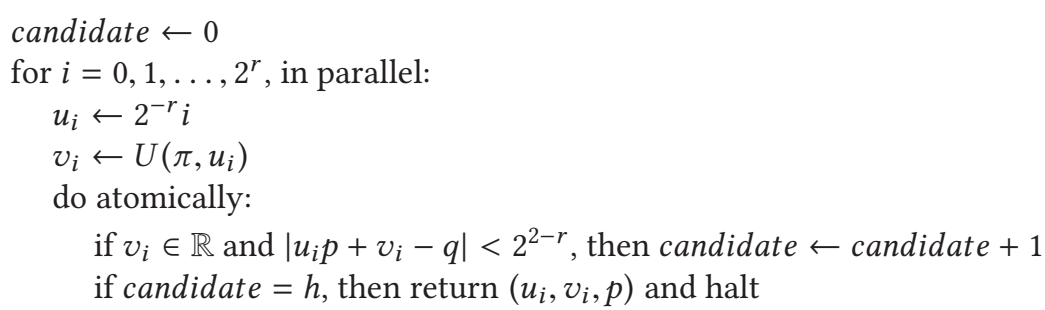


Fix $m \in[0,1]$ and $b \in \mathbb{R}$. For each $r \in \mathbb{N}$, let $m_{r}=2^{-r}\left\lfloor m \cdot 2^{r}\right\rfloor$, and fix $\pi_{r}$ testifying to the value of $\hat{K}_{r}\left(b \mid m_{r}\right)$ and $\sigma_{r}$ testifying to the value of $K_{r}(x, m x+b)$.

We complete the proof with the following four claims. Intuitively, Claim 1 says that no point in $B_{2^{-r}}(m)$ gives much less information about $b$ than $m_{r}$ does. Claim 2 states that there is always some value of $h$ that causes this machine to return the desired output. Claim 3 says that for almost every $x$, this value does not grow too quickly with $r$, and Claim 4 says that (5.1) holds for every $\operatorname{such} x$.

Claim 1. For every $r \in \mathbb{N}, K_{r}(b \mid m)=\hat{K}_{r}\left(b \mid m_{r}\right)+o(r)$.

Proof. $K_{r}(b \mid m) \geq \hat{K}_{r}\left(b \mid m_{r}\right)$ by definition, since $m_{r} \in B_{2^{-r}}(m)$.

Let $\hat{b} \in B_{2^{-r}}(b)$ be such that $K\left(\hat{b} \mid m_{r}\right)=\hat{K}_{r}\left(b \mid m_{r}\right)$. Then

$$
\left|\left(\hat{b}, m_{r}\right)-(b, m)\right| \leq \sqrt{2} \cdot 2^{-r}<2^{1-r},
$$

so

$$
K\left(\hat{b}, m_{r}\right) \geq K_{r-1}(b, m)=K_{r}(b, m)+o(r),
$$

by Corollary 3.9 of Reference [4].

Let $\mu$ testify to the value of $K_{r}(m)$, and let $\hat{m}=U(\mu)$. Then $|\hat{m}-m|<2^{-r}$, so $\left|\hat{m}-m_{r}\right|<2^{1-r}$. Thus, once $\hat{m}$ and $r$ have been specified, there are at most four possible values for $m_{r}$. Therefore there is a prefix Turing machine that takes as input $\mu$, an encoding of $r$ of length $o(r)$, and $O(1)$ additional bits and outputs $m_{r}$. We conclude that $K\left(m_{r}\right) \leq K_{r}(m)+o(r)$. Therefore, we have

$$
\begin{aligned}
\hat{K}_{r}\left(b \mid m_{r}\right) & =K\left(\hat{b} \mid m_{r}\right) \\
& =K\left(\hat{b}, m_{r}\right)-K\left(m_{r}\right)+o(r) \\
& \geq K_{r}(b, m)+o(r)-\left(K_{r}(m)+o(r)\right)+o(r) \\
& =K_{r}(b \mid m)+o(r),
\end{aligned}
$$

by Theorem 12 .

Claim 2. For each $x \in[0,1]$ and $r \in \mathbb{N}$, there exists an $h \in \mathbb{N}$ such that

$$
M\left(\rho \pi_{r} \sigma_{r} \eta\right) \in B_{2^{1-r}}(m, b, x),
$$

where $U(\rho)=r$ and $U(\eta)=h$.

Proof. Fix $x \in[0,1]$ and $r \in \mathbb{N}$. It is clear that for some $j \in\left\{0,1, \ldots, 2^{r}\right\},\left|u_{j}-m\right|<2^{-r}$. By the definition of $K_{r}(b \mid m), u_{j} \in \mathbb{Q} \cap B_{2^{-r}}(m)$ implies that $U\left(\pi_{r}, u_{j}\right)$ halts and outputs $v_{j} \in \mathbb{Q} \cap B_{2^{-r}}(b)$. $U\left(\sigma_{r}\right) \in B_{2^{-r}}(x, m x+b)$ by the definition of $\sigma_{r}$, so $|p-x|<2^{-r}$. It follows that

$$
\left|\left(u_{j}, v_{j}, p\right)-(m, x, b)\right|<\sqrt{3\left(2^{-r}\right)^{2}}<2^{1-r} .
$$

It remains to show that $\left|u_{i} p+v_{j}-q\right|<2^{2-r}$. To do so, we repeatedly apply the triangle inequality and use the fact that $x, m \in[0,1]$ :

$$
\begin{aligned}
\left|u_{i} p+v_{j}-q\right| & \leq\left|u_{i} p+v_{j}-(m x+b)\right|+|m x+b-q| \\
& <\left|u_{j} p-m x+v_{j}-b\right|+2^{-r} \\
& \leq\left|u_{j} p-m x\right|+\left|v_{j}-b\right|+2^{-r} \\
& <\left|u_{j} p-u_{j} x\right|+\left|u_{j} x-m x\right|+2^{1-r} \\
& \leq|p-x|+\left|u_{j}-m\right|+2^{1-r} \\
& <2^{2-r}
\end{aligned}
$$


For every $x \in[0,1]$ and $r \in \mathbb{N}$, define $h(x, r)$ to be the minimal $h$ satisfying the conditions of Claim 2.

Claim 3. For almost every $x \in[0,1], \log (h(x, r))=o(r)$.

Proof. By the countable additivity of Lebesgue measure, it suffices to show for every $k \in \mathbb{N}$ that the set

$$
D_{k}=\{x \in[0,1]: \exists \text { infinitely many } r \in \mathbb{N} \text { such that } \log (h(x, r))>r / k\}
$$

has Lebesgue measure 0 . For each $r \in \mathbb{N}$, let $D_{k, r}=\left\{x: h(x, r)>2^{r / k}\right\}$. We now estimate $\lambda\left(D_{k, r}\right)$, the Lebesgue measure of $D_{k, r}$.

For fixed $x$ and $r$, the algorithm run by the machine $M$ entails

$$
h(x, r) \leq\left|\left\{i:\left|u_{i} p+v_{i}-q\right|<2^{2-r}\right\}\right| .
$$

For fixed $i$,

$$
\begin{aligned}
\left|u_{i} p+v_{i}-q\right| & >\left|u_{i} x-u_{i} p\right|+\left|u_{i} p+v_{i}-q\right|-2^{-r} \\
& \geq\left|u_{i} x+v_{i}-q\right|-2^{-r} \\
& >\left|u_{i} x+v_{i}-q\right|+|q-(m x+b)|-2^{1-r} \\
& \geq\left|u_{i} x+v_{i}-(m x+b)\right|-2^{1-r} .
\end{aligned}
$$

That is,

$$
\left\{i:\left|u_{i} p+v_{i}-q\right|<2^{2-r}\right\} \subseteq\left\{i:\left|u_{i} x+v_{i}-(m x+b)\right|-2^{1-r}<2^{2-r}\right\},
$$

so

$$
h(x, r) \leq\left|\left\{i:\left|u_{i} x+v_{i}-(m x+b)\right|<2^{3-r}\right\}\right| .
$$

For fixed $r$ and $i=0,1, \ldots, 2^{r}$, define

$$
C_{i}^{r}=\left\{x \in[0,1]:\left|u_{i} x+v_{i}-(m x+b)\right|<2^{3-r}\right\},
$$

For each $i$, if $m=u_{i}$, then $C_{i}^{r}$ is either $[0,1]$ or empty; otherwise, $C_{i}^{r}$ is an interval of length

$$
\lambda\left(C_{i}^{r}\right) \leq \min \left\{\frac{2^{3-r}}{\left|u_{i}-m\right|}, 1\right\} .
$$

Notice that for each $k=0, \ldots, 2^{r}$, there are at most 2 values of $i$ for which

$$
2^{-r} k \leq\left|u_{i}-m\right|<2^{-r}(k+1),
$$

so we have

$$
\begin{aligned}
\int_{0}^{1} h(x, r) d x & \leq \sum_{i=0}^{2^{r}} \lambda\left(C_{i}^{r}\right) \\
& \leq 2+\sum_{k=1}^{2^{r}} 2 \frac{2^{3-r}}{2^{-r} k} \\
& =2+2^{4} \sum_{k=1}^{2^{r}} \frac{1}{k} \\
& <r 2^{6} .
\end{aligned}
$$

Thus, as $h(x, r)>2^{r / k}$ for all $x \in D_{k, r}$,

$$
\lambda\left(D_{k, r}\right)<\frac{r 2^{6}}{2^{r / k}}=r 2^{6-r / k}
$$


This implies that

$$
\sum_{r=1}^{\infty} \lambda\left(D_{k, r}\right)<\infty,
$$

so the Borel-Cantelli Lemma tells us that $\lambda\left(D_{k}\right)=0$.

Claim 4. For every $x \in[0,1]$, if $\log (h(x, r))=o(r)$, then

$$
\liminf _{r \rightarrow \infty} \frac{K_{r}(m, b, x)-K_{r}(b \mid m)}{r} \leq \operatorname{dim}(x, m x+b) .
$$

Proof. For fixed $r$, Claim 2 gives

$$
K_{r-1}(m, b, x) \leq K\left(u_{i}, v_{i}, p\right) \leq K_{M}\left(u_{i}, v_{i}, p\right)+c_{M},
$$

where $c_{M}$ is an optimality constant for $M$. Let $\rho$ and $\eta$ testify to the values of $K(r)$ and $K(h(x, r))$, respectively. Then $K_{M}\left(u_{i}, v_{i}, p\right) \leq\left|\rho \pi_{r} \sigma_{r} \eta\right|$. By our choices of $\rho, \pi_{r}, \sigma_{r}$, and $\eta$,

$$
\begin{aligned}
\left|\rho \pi_{r} \sigma_{r} \eta\right| & =K(r)+\hat{K}_{r}\left(b \mid m_{r}\right)+K_{r}(x, m x+b)+K(h(x, r)) \\
& =K(r)+K_{r}(b \mid m)+K_{r}(x, m x+b)+K(h(x, r))+o(r),
\end{aligned}
$$

by Claim 1. By Corollary 3.9 of Reference [4],

$$
\begin{aligned}
& \liminf _{r \rightarrow \infty} \frac{K_{r}(m, b, x)-K_{r}(b \mid m)}{r} \\
& \quad=\liminf _{r \rightarrow \infty} \frac{K_{r-1}(m, b, x)-K_{r}(b \mid m)+o(r)}{r} \\
& \quad \leq \liminf _{r \rightarrow \infty} \frac{K(r)+K_{r}(x, m x+b)+K(h(x, r))+o(r)}{r} \\
& \quad \leq \liminf _{r \rightarrow \infty} \frac{K_{r}(x, m x+b)}{r}+\limsup _{r \rightarrow \infty} \frac{K(r)+K(h(x, r))+o(r)}{r} \\
& \quad=\operatorname{dim}(x, m x+b)+\limsup _{r \rightarrow \infty} \frac{K(h(x, r))}{r} .
\end{aligned}
$$

Applying Observation 4, for some constant $c$,

$$
\begin{aligned}
\limsup _{r \rightarrow \infty} \frac{K(h(x, r))}{r} & \leq \limsup _{r \rightarrow \infty} \frac{\log (1+h(x, r))+2 \log \log (2+h(x, r))+c}{r} \\
& =\limsup _{r \rightarrow \infty} \frac{\log (h(x, r))+2 \log \log (h(x, r))}{r} .
\end{aligned}
$$

If $\log (h(x, r))=o(r)$, then this is

$$
\limsup _{r \rightarrow \infty} \frac{o(r)+2 \log (o(r))}{r}=0 .
$$

The lemma follows immediately from Claims 3 and 4 .

Proof of Theorem 16. Let $K$ be a Kakeya set in $\mathbb{R}^{2}$. By Theorem 1, there exists an oracle $A$ such that

$$
\operatorname{dim}_{H}(K)=\sup _{p \in K} \operatorname{dim}^{A}(p) .
$$

Let $m \in[0,1]$ such that $\operatorname{dim}^{A}(m)=1$; such an $m$ exists by Theorem 4.5 of Reference [22]. $K$ contains a unit line segment $L$ of slope $m$. Let $\left(x_{0}, y_{0}\right)$ be the left endpoint of such a segment. Let $q \in \mathbb{Q} \cap\left[x_{0}, x_{0}+1 / 8\right]$, and let $L^{\prime}$ be the unit segment of slope $m$ whose left endpoint is $\left(x_{0}-q, y_{0}\right)$. Let $b=y_{0}+m \cdot\left(q-x_{0}\right)$, the $y$-intercept of $L^{\prime}$. 
By a relativized version of Lemma 17 , there is some $x \in[0,1 / 2]$ such that $\operatorname{dim}^{A, m, b}(x)=1$ and

$$
\liminf _{r \rightarrow \infty} \frac{K_{r}^{A}(m, b, x)-K_{r}^{A}(b \mid m)}{r} \leq \operatorname{dim}^{A}(x, m x+b) .
$$

This holds because almost every $x \in[0,1 / 2]$ is algorithmically random relative to $(A, m, b)$ and hence satisfies $\operatorname{dim}^{A, m, b}(x)=1$.

Fix such an $x$, and notice that $(x, m x+b) \in L^{\prime}$. Now, applying a relativized version of Theorem 12 ,

$$
\begin{aligned}
\operatorname{dim}^{A}(x, m x+b) & \geq \liminf _{r \rightarrow \infty} \frac{K_{r}^{A}(m, b, x)-K_{r}^{A}(b \mid m)}{r} \\
& =\liminf _{r \rightarrow \infty} \frac{K_{r}^{A}(m, b, x)-K_{r}^{A}(b, m)+K_{r}^{A}(m)}{r} \\
& =\liminf _{r \rightarrow \infty} \frac{K_{r}^{A}(x \mid b, m)+K_{r}^{A}(m)}{r} \\
& \geq \liminf _{r \rightarrow \infty} \frac{K_{r}^{A}(x \mid b, m)}{r}+\liminf _{r \rightarrow \infty} \frac{K_{r}^{A}(m)}{r} .
\end{aligned}
$$

By Lemma $14, K_{r}^{A}(x \mid b, m) \geq K_{r}^{A, b, m}(x)+o(r)$, so we have

$$
\begin{aligned}
\operatorname{dim}^{A}(x, m x+b) & \geq \liminf _{r \rightarrow \infty} \frac{K_{r}^{A, b, m}(x)}{r}+\liminf _{r \rightarrow \infty} \frac{K_{r}^{A}(m)}{r} \\
& =\operatorname{dim}^{A, b, m}(x)+\operatorname{dim}^{A}(m),
\end{aligned}
$$

which is 2 by our choices of $m$ and $x$.

By Observation 6,

$$
\operatorname{dim}^{A}(x, m x+b)=\operatorname{dim}^{A}(x+q, m x+b) .
$$

Hence, there exists a point $(x+q, m x+b) \in K$ such that $\operatorname{dim}^{A}(x+q, m x+b) \geq 2$. By Theorem 1 , the point-to-set principle for Hausdorff dimension, this completes the proof.

It is natural to ask what prevents us from extending this proof to $\mathbb{R}^{n}$ for all $n \geq 2$. The point of failure in a direct extension would be Claim 3 in the proof of Lemma 17. Speaking informally, the problem is that the total number of candidates may grow as $2^{(n-1) r}$, meaning that $\log (h(x, r))$ could be $\Omega((n-2) r)$ for every $x$.

\section{CONCLUSION}

This article shows a new way in which theoretical computer science can be used to answer questions that may appear unrelated to computation. We are hopeful that our new proof of Davies's theorem will open the way for using constructive fractal dimensions to make new progress in geometric measure theory and that conditional dimensions will be a useful component of the information theoretic apparatus for studying dimension.

\section{ACKNOWLEDGMENTS}

We thank Eric Allender for useful corrections and five anonymous reviewers of this and an earlier version of this work for helpful input regarding presentation.

\section{REFERENCES}

[1] K. B. Athreya, J. M. Hitchcock, J. H. Lutz, and E. Mayordomo. 2007. Effective strong dimension in algorithmic information and computational complexity. SIAM f. Comput. 37, 3, 671-705.

[2] A. S. Besicovitch. 1919. Sur deux questions d'intégrabilité des fonctions. J. Soc. Phys. Math. l'Univ. Perm 2, 105-123. 
[3] A. S. Besicovitch. 1928. On Kakeya's problem and a similar one. Math. Zeitschr. 27, 312-320.

[4] A. Case and J. H. Lutz. 2015a. Mutual dimension. ACM Trans. Comput. Theory 7, 3, 12.

[5] A. Case and J. H. Lutz. 2015b. Mutual dimension and random sequences. In Mathematical Foundations of Computer Science, Lecture Notes in Computer Science, Vol. 9235. Springer, 199-210.

[6] G. J. Chaitin. 1966. On the length of programs for computing finite binary sequences. f. ACM 13, 4, 547-569.

[7] G. J. Chaitin. 1969. On the length of programs for computing finite binary sequences: statistical considerations. $\mathcal{F}$. ACM 16, 1, 145-159.

[8] T. R. Cover and J. A. Thomas. 2006. Elements of Information Theory (2nd ed.). Wiley.

[9] R. O. Davies. 1971. Some remarks on the Kakeya problem. Proc. Cambr. Phil. Soc. 69, 417-421.

[10] R. Dougherty, J. H. Lutz, R. D. Mauldin, and J. Teutsch. 2014. Translating the Cantor set by a random real. Trans. Am. Math. Soc. 366, 3027-3041.

[11] R. Downey and D. Hirschfeldt. 2010. Algorithmic Randomness and Complexity. Springer-Verlag.

[12] Z. Dvir. 2009. On the size of Kakeya sets in finite fields. F. Am. Math. Soc. 22, 1093-1097.

[13] K. Falconer. 2014. Fractal Geometry: Mathematical Foundations and Applications (3rd ed.). Wiley.

[14] X. Gu, J. H. Lutz, and E. Mayordomo. 2006. Points on computable curves. In Proceedings of the IEEE Symposium on Foundations of Computer Science (FOCS'06). IEEE, Los Alamitos, CA, 469-474.

[15] X. Gu, J. H. Lutz, E. Mayordomo, and P. Moser. 2014. Dimension spectra of random subfractals of self-similar fractals. Ann. Pure Appl. Logic 165, 11, 1707-1726.

[16] F. Hausdorff. 1919. Dimension und äusseres Mass. Math. Ann. 79, 157-179.

[17] A. N. Kolmogorov. 1965. Three approaches to the quantitative definition of information. Probl. Inf. Transmiss. 1, 1, 1-7.

[18] L. A. Levin. 1973. On the notion of a random sequence. Sov. Math Dokl. 14, 5, 1413-1416.

[19] L. A. Levin. 1974. Laws of information conservation (nongrowth) and aspects of the foundation of probability theory. Probl. Pered. Inf. 10, 3, 30-35.

[20] M. Li and P. M. Vitányi. 2008. An Introduction to Kolmogorov Complexity and Its Applications (3rd ed.). Springer.

[21] J. H. Lutz. 2003a. Dimension in complexity classes. SIAM J. Comput. 32, 5, 1236-1259.

[22] J. H. Lutz. 2003b. The dimensions of individual strings and sequences. Inf. Comput. 187, 1, 49-79.

[23] J. H. Lutz and N. Lutz. 2015. Lines missing every random point. Computability 4, 2, 85-102.

[24] J. H. Lutz and E. Mayordomo. 2008. Dimensions of points in self-similar fractals. SIAM J. Comput. 38, 3, $1080-1112$.

[25] J. H. Lutz and K. Weihrauch. 2008. Connectivity properties of dimension level sets. Math. Logic Quart. 54, 483-491.

[26] J. M. Marstrand. 1954. Some fundamental geometrical properties of plane sets of fractional dimensions. Proc. Lond. Math. Soc. 4, 3, 257-302.

[27] P. Martin-Löf. 1966. The definition of random sequences. Inf. Contr. 9, 6, 602-619.

[28] E. Mayordomo. 2002. A Kolmogorov complexity characterization of constructive Hausdorff dimension. Inf. Process. Lett. 84, 1, 1-3.

[29] A. Nies. 2009. Computability and Randomness. Oxford University Press, New York, NY.

[30] C. E. Shannon. 1948. A mathematical theory of communication. Bell Syst. Techn. 7. 27, 3-4, 379-423, 623-656.

[31] R. J. Solomonoff. 1964. A formal theory of inductive inference. Inf. Contr. 7, 1-2, 1-22, 224-254.

[32] E. M. Stein and R. Shakarchi. 2005. Real Analysis: Measure Theory, Integration, and Hilbert Spaces. Princeton Lectures in Analysis. Princeton University Press, Princeton, NJ.

[33] T. Tao. 2000. From rotating needles to stability of waves: emerging connections between combinatorics, analysis, and PDE. Not. Am. Math. Soc. 48, 294-303.

[34] A. M. Turing. 1937. On computable numbers, with an application to the Entscheidungsproblem. A correction. Proc. Lond. Math. Soc. 43, 2, 544-546.

[35] K. Weihrauch. 2000. Computable Analysis: An Introduction. Springer.

[36] T. Wolff. 1996. Recent work connected with the Kakeya problem. In Prospects in Mathematics. American Mathematical Society, Princeton, NJ, 129-162.

Received February 2017; revised December 2017; accepted January 2018 